\title{
Verantwortung verantworten
}

\section{BRUNO STAFFELBACH ${ }^{*}$}

Korreferat zum Beitrag von Susan Beyer, Stephan Bohn, Toni Grünheid, Sebastian G. M. Händscbke, Raluca Kerekes, Jonas C. Müller, Peter Walgenbach

\section{Das Problem}

Menschen haben ein Grundverständnis davon, was moralisch legitim und nicht legitim ist, oder was gerecht, fair und billig ist. Es ist ihnen ein Bedürfnis, in einem moralisch guten Licht dazustehen. Das moralische Schamgefühl ist eine Emotion, mit der man bereits von früher Kindheit an umzugehen lernt - die Einen so, die Anderen anders. Auch in Unternehmen motiviert moralisches Handeln oder es frustriert, es begeistert oder es kostet die Gefolgschaft. Eine Analyse aus Anwaltskanzleien zeigte zum Beispiel (vgl. Kammeyer-Mueller et al. 2012), dass bei Anwältinnen und Anwälten mit moralischen Konflikten - etwa bei Pflichtmandaten, die sie mit ihrem Gewissen nicht vereinbaren konnten - die emotionale Erschöpfung stieg und die Laufbahnzufriedenheit abnahm. Dies führte dazu, dass sie eher kündigten und neue Karriereoptionen suchten.

Moral wird damit ökonomisch relevant. Moralische Vorstellungen aber sind vielfältig. Aus einem breiten Sortiment können heute alle ihr eigenes Moral-Portfolio zusammenstellen. Moral kann man kaufen („faire Preise!"), ansparen („ethische Fonds!") und kompensieren (,spende für eine NGO!" ). Moral wird zu einem Werbemittel für Produkte, Leistungen und Arbeitsplätze. Mit Moral kann man sich profilieren. Moral wird zum Argument im politischen Prozess, zum strategischen Zug, zum rhetorischen Mittel. Hier setzt die explorative Studie von Susan Beyer, Stephan Bohn, Toni Grünheid, Sebastian Händschke, Raluca Kerekes, Jonas Müller und Peter Walgenbach an. Sie wollen herausfinden, wofür Unternehmungen in einem Kontext moralischer Diversität Verantwortung reklamieren.

\section{Der Befund}

Die Autoren analysieren die Texte im Internetauftritt von E.ON, Daimler und der Deutschen Bank. Sie stellen fest, dass diese drei DAX30-Unternehmungen in den Selbstbeschreibungen auf ihren Websites vorgeben, gegenüber verschiedenen Akteuren, Prinzipien und Interessen Verantwortung zu übernehmen, dass sie sich dabei u.a. auch auf verschiedene moralische und politische Autoritäten beziehen und dass sie über alle Verantwortungsinhalte hinweg dem „Ökonomischen i.e.S.“ relativ wenig Gewicht einräumen. Gemessen am Vokabular der analysierten Websites der drei Fir-

Prof. Dr. Bruno Staffelbach, Institut für Betriebswirtschaftslehre, Universität Zürich, Plattenstrasse 14, CH-8032 Zürich, Tel.: +41 4463429 96, Fax: +41 4463449 15, E-Mail: bruno.staffelbach@business.uzh.ch, Forschungsschwerpunkte: Human Resource Management, Management-Ethik. 
men kommen die Autoren deshalb zum Schluss, dass „die Betrachtung von Unternehmungen als Einrichtungen, die ausschliesslich der Erzielung eines Profits dienen, deutlich zu kurz greift" (S. XXX). Sie reihen sich damit in einen aktuellen wirtschaftswissenschaftlichen Diskurs ein, der in der Wirtschaft mehr erkennt als „rein ökonomische“ oder monetäre Phänomene. Deshalb sei die Wirtschaftswissenschaft mit anderen Bereichen wie etwa der Psychologie und/oder der Soziologie zu verknüpfen.

\section{Die Beurteilung}

\subsection{Methodische und ontologische Reduktion}

Keine Wissenschaft, die sich mit lebenden Systemen befasst, kann diese in ihrer ganzen Vielfalt und Dynamik abbilden. Solche Wissenschaften kommen deshalb nicht darum herum, die Komplexität ihres Gegenstandsbereiches auf geeignete Art und Weise zu reduzieren, zum Beispiel durch Vereinfachung, durch Verallgemeinerung, oder durch Konzentration auf Einzelteile. Die Ökonomik ist voll von solchen methodisch bedingten Reduktionen. Der Homo oeconomicus, die ceteris-paribus-Bedingung oder das Geld als Messgrösse sind Beispiele dafür. Umsätze, Kosten, Cashflow, Aktienkurse oder Kapitalrenditen etwa sind betriebswirtschaftliche Geldgrössen. Ihr Informationswert ist hoch, wenn man die Bedingungen kennt, unter denen sie zustande gekommen sind. Wie beim Sport in Teilsekunden an der Ziellinie zeigt sich in dieser Sicht der betriebswirtschaftliche Erfolg letztlich als Geldgrössen in der Bilanz, in der Erfolgsrechnung oder allenfalls an der Börse. Aber eben: der betriebswirtschaftliche Erfolg zeigt sich letztlich in Euros, Dollars oder Franken. Problematisch wird es, wenn die Welt des Wirtschaftens ausschliesslich in Euros, Dollars oder Franken wahrgenommen, reflektiert und beurteilt wird. Dann nämlich wird Ökonomik auf Monetik reduziert, dann wird eine Partialanalyse zu einer Totalbetrachtung und dann tritt an die Stelle einer (ursprünglich) methodischen Reduktion eine ontologische Reduktion.

Nimmt man die Kommunikation auf den Websites der Unternehmungen als Basis, dann greift die Annahme, dass sich eine Unternehmung über ihre Gewinnfunktion abbilden lässt, tatsächlich zu kurz. Real sind Unternehmungen offenbar mehr als nur „Maschinerien“, „Organismen“ oder „Verbünde“ zur Erzielung maximaler Gewinne. Es überrascht deshalb nicht, dass in der Analyse der Internetauftritte dieser drei Unternehmungen Verantwortungsinhalte und -qualitäten erkennbar werden, die über das "Ökonomische“ hinausreichen. Vorsicht ist dabei allerdings bei der Interpretation der Häufigkeiten einzelner Aspekte von Verantwortung geboten. Es kann verschiedene Gründe haben, weshalb ein Aspekt X mehr oder weniger häufig genannt wird: er ist selbstverständlich, weshalb er nicht genannt wird, er ist prekär und knapp, weshalb er häufig genannt wird oder er ist nicht knapp, sondern in aller Leute Munde und wird deshalb oft genannt. In einer Folgeuntersuchung könnten hier Defizitwahrnehmungen, kommunikative Kontexte und dominierende „Sprachspiele“ untersucht werden.

\subsection{Worte und Taten}

Wir leben in einer Leistungsgesellschaft. Eine Leistungsgesellschaft erhebt das Leistungsprinzip zum Grundsatz Prinzip sozialer Verteilungsgerechtigkeit. Wenn man an alternative Prinzipien von anderen Gesellschaften denkt - Geburt, Konfession, Ein- 
kommen der Eltern, politische Zugehörigkeit - so hat dieses Prinzip seine Vorteile. Die zentrale Frage, die sich damit aber verbindet, lautet: was heisst Leistung? Man spricht über die Leistung von Mutter Theresa, von Albert Einstein oder von Wolfgang Amadeus Mozart. Drogendealer, Geldwäscher oder die Mafia haben auch ihre Vorstellungen von Leistung. 'Leistung' per se ist offen. Es ist ein Wort, das mit unterschiedlichen Inhalten gefüllt werden kann. Es ist ein Hülsenwort. Mit ,Verantwortung' verhält es sich ähnlich. Es ist ein Verdienst der vorliegenden Studie, das Wort ,Verantwortung' im Kontext der Unternehmungskommunikation empirisch-induktiv über verschiedene Kategorien analysiert und präzisiert zu haben. Im Zentrum der Analyse steht dabei das Vokabular, mit dem Verantwortung deklariert wird.

,Verantwortung' ist ein Kommunikationsbegriff (vgl. Saladin 1984: 30). Er beinhaltet ein „Rede und Antwort geben“ von einem Verantwortungsträger - der Unternehmung als Akteur - gegenüber bzw. „,vor einer Instanz, die Rechenschaft fordert" (Höffe 1986: 263). ,Verantwortung' in diesem Sinne hat also einerseits einen deklarativen Aspekt. Andererseits geht es bei ,Verantwortung' um ein Einstehen für das Tun oder Lassen einer Handlung. ,Verantwortung' hat somit auch einen aktionalen Aspekt. Damit ist die Verpflichtung verbunden, allfällige Folgen, Konsequenzen oder Sanktionen der Handlung zu tragen.

\subsection{Ethik und Verantwortung}

Unternehmungen operieren nicht in einem wertfreien Raum. Unternehmungen kommen also nicht darum herum, für sie relevante Werte und Normen zu bestimmen (vgl. Staffelbach 2012). Hinzu kommt, dass Märkte unabhängig davon funktionieren, ob es sich um Märkte für Kaugummis, Autos, Psychopharmaka oder Tretminen handelt. Betriebswirtschaftlichen Instrumenten und Methoden ist es gleichgültig, ob sie im Roten Kreuz, der Auto- oder Bankindustrie, der Prostitution oder für karitative Zwecke eingesetzt werden. In der Ökonomik geht es um Mittel. Die Zwecke sind offen. Weil es in der Ökonomik um Mittel geht, die für beliebige Zwecke eingesetzt werden können, benötigt man etwas, um diese der Beliebigkeit zu entziehen.

Unabhängig davon zeigen sich bei der Unternehmungsführung verschiedene Spannungsfelder. Einerseits gibt es beispielsweise die Gebote einer ideal gedachten Marktökonomie, wie sie auf Adam Smith zurückgehen: sorge für vollständige Information, respektiere die Konsumentensouveränität, verhindere Monopole, halte die Märkte offen und vermeide negative externe Effekte in Raum und Zeit. Andererseits steht in vielen Lehrbüchern zur strategischen Unternehmungsführung: nutze Informationen zu Deinem Vorteil, achte auf den Shareholder-Value, entwickle und bündle Kernkompetenzen um Monopolrenten abzuschöpfen, segmentiere Märkte, baue Eintrittsbarrieren und mache Kosten-Nutzen-Rechnungen mit zeitdiskontierten Grössen. So sind Konflikte vorprogrammiert. In diesem „Geflecht“ von widersprüchlichen Vorstellungen braucht es Referenzpunkte und Orientierungen.

Gesucht sind also Normen, die das Unternehmungshandeln geistig, inhaltlich und zeitlich konkretisieren und koordinieren. Sie sind Grundlage zur Bildung einer eigenen moralischen Unternehmungsidentität. In der Flut unterschiedlicher Moralvorstellungen und weil Moral auch strategisch genutzt werden kann, braucht es Qualitätsmassstäbe. Dazu braucht es ethische Theorien. Die Studie von Susan Beyer, Stephan Bohn, 
Toni Grünheid, Sebastian Händschke, Raluca Kerekes, Jonas Müller und Peter Walgenbach ist ein Start zu einer deskriptiven Unternehmungsethik zum Verantworten von Verantwortung.

\section{Literaturverzeichnis}

Höffe, O. (1986): Verantwortung, in: Lexikon der Ethik, München: Beck, 263-264.

Kammeyer-Mueller, J. D./Simon, L. S./Rich, B. L. (2012): The Psychic Cost of Doing Wrong: Ethical Conflict, Divestiture Socialization, and Emotional Exhaustion, in: Journal of Management, Vol. 38/No. 3, 784-808.

Saladin, P. (1984): Verantwortung als Staatsprinzip, Bern: Haupt.

Staffelbach, B. (2012): Betriebswirtschaftliche Führungsphilosophien. Politische Studien Nr. 449, München: Hans Seidel Stiftung. 